\title{
Cardiovascular disease risk among breast cancer survivors: an evolutionary concept analysis
}

This article was published in the following Dove Press journal:

Nursing: Research and Reviews

3 February 2017

Number of times this article has been viewed

\author{
Jacqueline B Vo' \\ Timiya S Nolan' \\ David E Vance' \\ Patricia A Patrician ${ }^{2}$ \\ Karen Meneses' \\ 'Office of Research and Scholarship, \\ ${ }^{2}$ Department of Family, Community \\ Health, and Systems, University of \\ Alabama at Birmingham School of \\ Nursing, Birmingham, AL, USA
}

Correspondence: Jacqueline B Vo Office of Research and Scholarship, University of Alabama at Birmingham School of Nursing, University Boulevard, 1720 2nd Avenue South, MT 504, Birmingham, AL 35294, USA

$\mathrm{Tel}+\mathrm{I} 2059347580$

$\mathrm{Fax}+\mathrm{I} 2059756194$

Email jacbui@uab.edu
Background: More than 3.5 million breast cancer survivors are living in the US, and the overall five-year survival rate is approaching $90 \%$. With increased survival and cancer treatment-related cardiotoxicities, there has been a rise in cardiovascular diseases among breast cancer survivors. Yet, cardiovascular disease risk among breast cancer survivors has not been well conceptualized. The purpose of this article was to analyze and define the concept of cardiovascular disease risk among breast cancer survivors.

Methods: The databases CINAHL, EMBASE, and PubMed were used to identify articles that explored cardiovascular disease risk among breast cancer survivors. The search yielded 357 articles, which were reviewed for eligibility. Thirty articles were selected based on the inclusion/ exclusion criteria. The concept of cardiovascular disease risk among breast cancer survivors was analyzed using Rodgers' evolutionary concept analysis method.

Results: The analysis suggests that cardiovascular disease risk among breast cancer survivors consists of several attributes: cancer treatment (chemotherapy, targeted therapies, radiation therapy, and endocrine therapy), modifiable risk factors (obesity, physical inactivity, poor diet, and smoking), and nonmodifiable risk factors (age, family history, and race). The antecedent identified includes breast cancer diagnosis and the consequence identified includes the development of cardiovascular disease.

Conclusion: Findings suggest the need for increased education and understanding of cardiovascular disease risk among health care providers and patients. Survivorship care plans can incorporate cardiovascular disease risk monitoring and screening. Future research is needed to explore interventions and develop stratified screening guidelines for breast cancer survivors.

Keywords: breast cancer survivors, cardiovascular disease risk, Rodgers' evolutionary concept analysis method, cancer survivorship, cardiotoxicity

\section{Introduction}

Cardiovascular diseases are the leading cause of death in the US and account for more than 600,000 deaths each year. ${ }^{1}$ The American Heart Association has set a goal to reduce cardiovascular diseases by $20 \%$ by the year $2020 .^{2}$ Cancer survivors comprise a population who are at an increased risk of cardiovascular disease. Advances in cancer treatment have contributed to survivors living longer; yet, many cancer treatments are associated with lifelong cardiovascular disease risk. ${ }^{2-5} \mathrm{As}$ a result of this combination of factors, a new field called cardio-oncology has emerged, which is focused on the treatment of patients with cardiovascular-related events after cancer.

The largest group of cancer survivors in the US comprises breast cancer survivors. As of 2016, approximately 3.5 million breast cancer survivors are living in 
the US. ${ }^{6}$ Ninety percent of women diagnosed with breast cancer will survive for at least 5 years after diagnosis, ${ }^{7}$ shifting the cause of death from breast cancer to other agerelated conditions such as cardiovascular diseases. ${ }^{8}$ Breast cancer survivors are at an increased risk of cardiovascular diseases due to adverse effects from cancer treatment, such as cardiotoxicity, which may persist from the time of treatment to survivorship. ${ }^{9}$

Despite studies exploring cardiovascular disease risk among breast cancer survivors, guidelines for monitoring and reducing cardiovascular disease risk do not exist currently for patients treated in the US. Several leading organizations have developed general survivorship guidelines for breast cancer survivors, including the American Society of Clinical Oncology, the American Cancer Society, and the National Comprehensive Cancer Network. The American Society of Clinical Oncology and the American Cancer Society guidelines recommend cardiovascular disease risk monitoring as needed, similar to the general population, despite the higher risk of cardiovascular diseases in breast cancer survivors. ${ }^{9}$ The National Comprehensive Cancer Network recommends baseline cardiac function assessment for all cancer survivors treated with anthracycline chemotherapy $;{ }^{10}$ however, other chemotherapies and cancer treatments may contribute to an increased cardiovascular disease risk. ${ }^{9}$ Furthermore, many breast cancer survivors may experience symptoms of cardiovascular diseases years after treatment or may be asymptomatic. ${ }^{2-5}$ Yet, guidelines do not recommend long-term cardiac monitoring. While cardiotoxicity is addressed in these survivorship guidelines, recommendations for cardiovascular disease risk monitoring are not addressed.

In contrast to the US guidelines, the European Society for Medical Oncology recommends that patients treated with anthracyclines have their cardiac function measured at baseline prior to administration of anthracycline treatment, and 4 and 10 years after anthracycline treatment is completed. ${ }^{11}$ The guidelines also take into account the effects of different cancer treatments on cardiovascular disease risk including targeted and radiation therapies. Conforming to cardiovascular disease risk guidelines developed for the general population, as recommended by many American oncology organizations, may not be appropriate for breast cancer survivors due to their additional cancer treatment-related risk factors. Understanding the synergistic effects of cancer treatments combined with preexisting modifiable and nonmodifiable risk factors is crucial to defining the concept of cardiovascular disease risk among breast cancer survivors.
To facilitate our understanding of risk, a concept analysis using Rodgers' evolutionary concept analysis method was conducted on cardiovascular disease risk among breast cancer survivors. Cardiovascular disease risk factors in the general population are well known; however, cardiovascular disease risk among breast cancer survivors requires further clarification. The purpose of this concept analysis is to define cardiovascular disease risk for breast cancer survivors. First, the significance and background of cardiovascular disease risk among breast cancer survivors are reviewed. Next, the method of conducting Rodgers' evolutionary concept analysis on this topic is described. The results are examined following Rodgers' method. Finally, from the knowledge derived from this concept analysis, implications for nursing are provided.

\section{Methods \\ Concept analysis method}

The Rodgers' evolutionary concept analysis method was selected for three reasons. First, it uses an inductive approach to develop the concept of cardiovascular disease risk among breast cancer survivors. Second, it applies a rigorous six-step method. Third, concepts are viewed as cyclical and continuously evolving. The six steps in Rodgers' concept analysis include the following: 1) identifying the concept and associated terms, 2) selecting an appropriate setting or sample for data collection, 3 ) collecting data to identify the attributes of the concept, 4) analyzing the characteristics of the concept (i.e., surrogate terms, related concepts, antecedents, and consequences), 5) identifying an exemplar of the concept, and 6) identifying hypotheses and implications for future development. ${ }^{12}$ The identified concepts may be continually refined and synthesized as newer innovative research emerges. ${ }^{12}$

\section{Literature search method}

A literature search was conducted on July 22, 2016, using the databases PubMed, EMBASE, and CINAHL. The key terms used to search the databases included "breast cancer OR breast neoplasm" AND "survivors" AND “cardiovascular disease risk OR cardiotoxicity". The articles published only in English were chosen for this literature review. The results yielded 357 articles; duplicates were removed, resulting in 293 articles. Articles were included if published within the past 15 years and full text was available, excluding 106 articles. The remaining 187 articles were assessed for inclusion criteria: primary studies with breast cancer survivors among the population and cardiovascular disease risk were explored. Twenty-one articles met the inclusion criteria. Ascendancy (searching forward to articles that have cited 


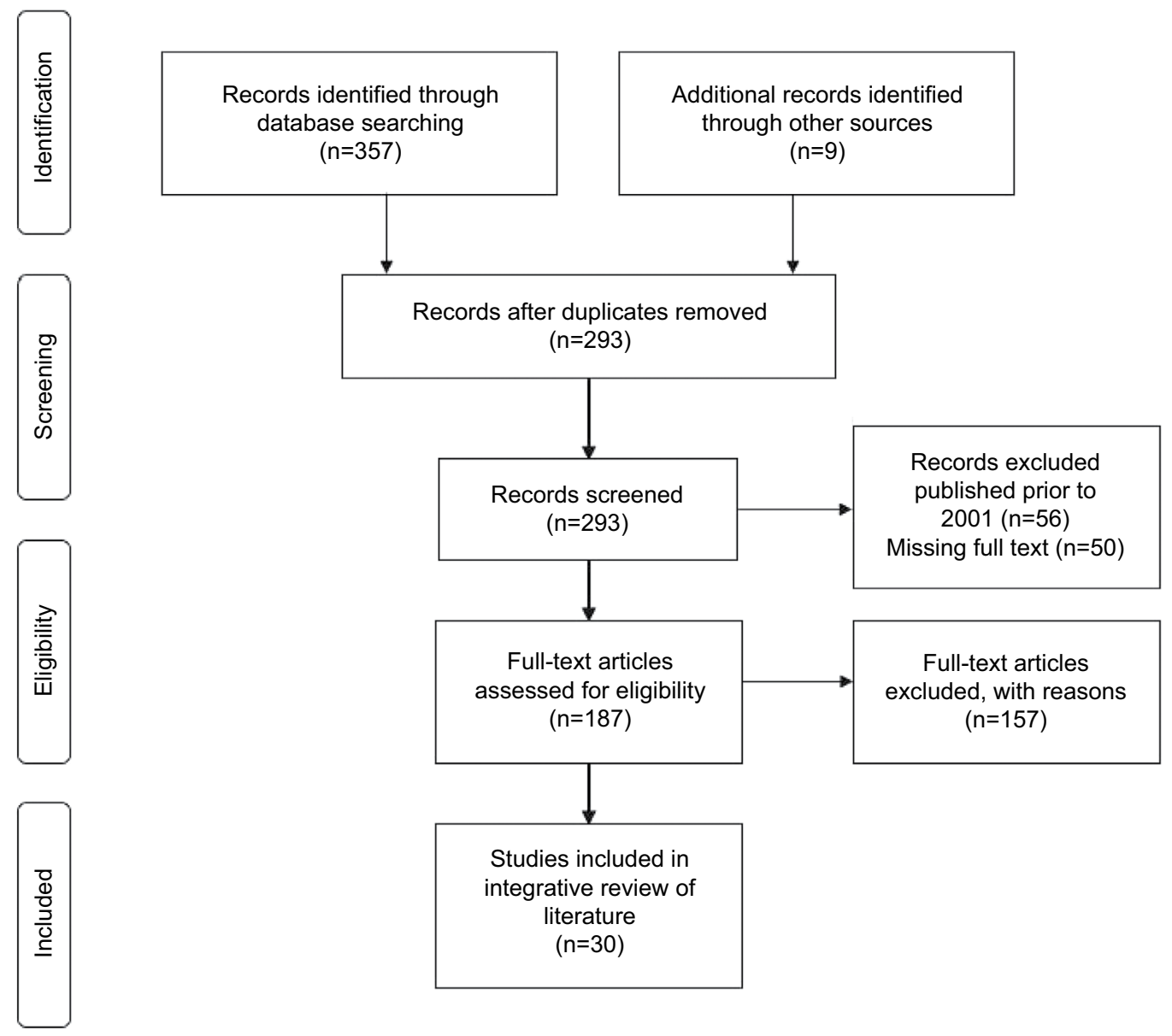

Figure I PRISMA flow diagram demonstrates screening method for articles.

Note: Copyright: @ 2009. Adapted from Moher D, Liberati A, Tetzlaff J, Altman DG; The PRISMA Group. Preferred Reporting Items for Systematic Reviews and MetaAnalyses: The PRISMA Statement. PLoS Med. 2009;6(7):eI000097. doi:10.137I/journal.pmed I000097.47

Abbreviation: PRISMA, Preferred Reporting Items for Systematic Reviews and Meta-Analysis.

selected articles) and descendancy (exploring articles used in the reference lists) approaches were used to identify nine additional articles that met the inclusion criteria, resulting in a total of 30 articles for this review. Figure 1 shows a Preferred Reporting Items for Systematic Reviews and Meta-Analysis (PRISMA) diagram describing the search strategy. Data were collected using the sample of 30 articles to conduct this concept analysis. The articles were reviewed to identify the attributes of the concept of cardiovascular disease risk among breast cancer survivors.

\section{Results}

Several themes were identified during the literature search and data analysis: conceptual definitions, operational definitions, and attributes of cardiovascular disease risk. The following sections outline findings from the 30 articles.

\section{Conceptual definitions}

Conceptual definitions include the necessary components of the concept or how it is scientifically understood. ${ }^{13}$ During the review of literature, conceptual definitions of cardiovascular disease risk were identified as follows: 1) incidence of cardiovascular diseases, 2) presence of cardiovascular disease risk factors, and 3) changes in heart function. Cardiovascular disease risk was often conceptually defined as incidence of cardiovascular diseases including ischemic heart disease, congestive heart failure, myocardial infarction, coronary artery disease, stroke, angina, atrial fibrillation, paroxysmal tachycardia, nonrheumatic valve disease, cardiomyopathy, aortic aneurysm, and arteriosclerosis..$^{3-4,8,14-29}$ While one study included angina as a cardiovascular disease risk factor, ${ }^{15}$ Darby et al indicated that angina would not be included as a risk factor due to difficulty with clinical identification of angina. ${ }^{19}$ Other investigators conceptually defined cardiovascular disease risk as the presence of cardiovascular disease risk factors including smoking and having an overweight or obese body mass index..$^{23,30,31}$ Changes in heart function, including decreases in left ventricular ejection fraction, presence of serum biomarkers, and changes in cardiorespiratory fitness, also comprised the conceptual 
definition of cardiovascular disease risk..$^{3,32-40}$ Three studies associated decreases in left ventricular ejection fraction with anthracycline chemotherapy and conceptually defined cardiovascular disease risk in breast cancer survivors as anthracycline-induced cardiotoxicity. 4,29,37

\section{Operational definition}

Operational definitions specify how the concept is measured or applied. ${ }^{12}$ Cardiovascular disease risk was operationalized using multiple methods throughout the literature. Incidence of cardiovascular diseases using morbidity and mortality was a common measure of cardiovascular disease risk among

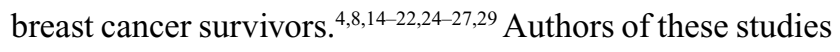
used electronic health records, large databases, and/or linkages to death indexes as data sources. Physical measurements of cardiovascular disease risk are ideal to reduce self-report bias; however, fewer studies conducted physical measurements of cardiovascular disease risk..$^{3,32-40}$ Four studies used self-reported history of cardiovascular diseases or cardiovascular disease risk factors as an operational definition. ${ }^{23,28,30,31}$

\section{Attributes}

According to Rodgers, attributes provide a "real definition as opposed to a nominal or dictionary definition". ${ }^{12}$ Attributes are the features that comprise the concept as opposed to the conceptual definition of the concept. The attributes of cardiovascular disease risk in breast cancer survivors are categorized as cancer treatment, modifiable risk factors, and nonmodifiable risk factors.

\section{Cancer treatment}

Chemotherapy, targeted therapies, radiation, and endocrine therapy are common cancer treatments associated with cardiotoxicity in breast cancer survivors. Chemotherapy agents used to treat breast cancer, such as anthracyclines and taxanes, were associated with heart failure and ischemic heart disease. ${ }^{15,17,24,38}$ The mechanism of action for anthracyclines, including epirubicin and doxorubicin, may lead to the production of free radicals. Anthracyclines were associated with the development of cardiomyopathy, arrhythmias, aortic stiffness, and heart failure. $3,4,35,39,40$ Cardiotoxic symptoms from anthracycline therapy may either appear early on in survivorship, such as within the first or second year, or have a late onset many years later. ${ }^{3,4}$ In addition, the anthracycline drug class is dose dependent; therefore, an increase in dosage is associated with increased risk of heart failure. ${ }^{3}$ Taxanes are often used as adjuvant therapy to anthracyclines in cancer treatment. When both drug classes were used concurrently, patients were at an increased risk of asymptomatic bradycardia. ${ }^{39}$ Targeted therapies, including trastuzumab, used with anthracycline-based chemotherapy demonstrated increased cardiovascular disease risk, including the development of heart failure and cardiomyopathy. ${ }^{16,20}$ Radiation therapy was associated with increased cardiovascular disease risk. ${ }^{14,19,22,24}$ Radiation to the left breast increases radiation exposure to the heart, leading to cardiac ischemia or damage to the coronary arteries. ${ }^{19}$ Endocrine therapies including aromatase inhibitors were also associated with an increased cardiovascular disease risk. ${ }^{28}$ In summary, the attribute of cancer treatment is associated with cardiovascular disease development and facilitates the definition of cardiovascular disease risk among breast cancer survivors.

\section{Modifiable factors}

Modifiable factors including obesity, physical inactivity, poor diet, and smoking may result in increased cardiovascular disease risk among breast cancer survivors. A body mass index greater than $25 \mathrm{~kg} / \mathrm{m}^{2}$ is associated with increased cardiovascular disease risk. ${ }^{2}$ After diagnosis, many breast cancer survivors gain weight and report higher body mass index as a result of cancer treatment and/or sedentary lifestyles. ${ }^{33}$ Obese breast cancer survivors are at higher cardiovascular disease risk compared with survivors with normal body mass index. ${ }^{27}$ Breast cancer survivors can lose weight and decrease body mass index through exercise, and physical activity has shown to be protective against developing increased cardiovascular disease risk. ${ }^{32}$ Yet, in a study exploring cardiorespiratory fitness, the investigators found that breast cancer survivors had a low cardiorespiratory fitness level that may impair the ability to exercise. ${ }^{32}$ Thus, breast cancer survivors who are unable to or lack motivation to exercise may be physically inactive, resulting in increased cardiovascular disease risk. Dyslipidemia, which may result from poor diets high in cholesterol, was identified as a risk factor for cardiovascular diseases and prevalent among cancer survivors. ${ }^{18}$

Smoking was associated with decreases in heart function leading to an increased cardiovascular disease risk in breast cancer survivors treated with chemotherapy. ${ }^{37}$ In combination with physical inactivity, obesity, and diet, smoking may lead to increased cardiovascular disease risk, particularly atherosclerosis and diminished function of blood vessels. ${ }^{41}$ Cancer survivors were also more likely to be smokers ${ }^{30,34}$ and less likely to be counseled on diet by health care providers. ${ }^{30}$ Therefore, modifiable risk factors and poor lifestyle choices can ultimately contribute to an increased cardiovascular disease risk among breast cancer survivors. 


\section{Nonmodifiable factors}

Nonmodifiable personal characteristics such as age and race can contribute to an increased cardiovascular disease risk among breast cancer survivors. Several studies demonstrated that as aging increases, cardiovascular disease risk increases regardless of previous breast cancer diagnosis. ${ }^{21,23,25}$ Older age among breast cancer survivors increased the risk of early

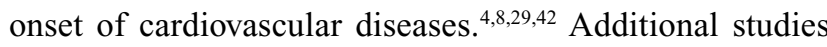
indicated that African American breast cancer survivors were at an increased cardiovascular disease risk compared to other races. ${ }^{23,31,36}$

Having a first-degree family history of heart disease also increased the cardiovascular disease risk. ${ }^{43}$ None of the reviewed articles explored family history as a cardiovascular disease risk factor. Figure 2 demonstrates the relationship between the concept and its attributes. Collectively, these studies support that the attributes of cancer treatments and modifiable and nonmodifiable risk factors contribute to the concept of cardiovascular disease risk among breast cancer survivors. Although family history was not explored in the selected articles, it is a known risk factor for cardiovascular diseases and is included in Figure 2.

\section{Characteristics of the concept}

Characteristics that describe the concept include the following: 1) providing surrogate terms and related concepts and
2) describing antecedents and consequences of the concept. ${ }^{12}$ The identified characteristics are outlined respectively.

\section{Surrogate terms and related concepts}

Surrogate terms are used interchangeably to express the concept. ${ }^{12}$ The term "cardiovascular disease risk" was not used consistently throughout the reviewed literature. The terms "cardiotoxicity", "cardiovascular disease development", and "incidences of cardiovascular disease" were used instead of "cardiovascular disease risk". The term "breast cancer survivors" was used consistently with several instances of "patient" instead of "survivors". Related concepts are similar to the concept but do not possess the same attributes. ${ }^{12}$ Since modifiable and nonmodifiable risk factors are attributes to both cardiovascular disease risk in the general population and the breast cancer population, "cardiovascular disease risk in the general population" is a related concept that shares similar but not all the same attributes.

\section{Antecedents and consequences}

Antecedents are events leading to the concept of interest. ${ }^{12}$ The antecedent to cardiovascular disease risk among breast cancer survivors is breast cancer diagnosis because patients become "survivors" at diagnosis. ${ }^{44}$ Additionally, consequences are events that follow as a result of the concept's occurrence. ${ }^{12}$ Breast cancer survivors may develop

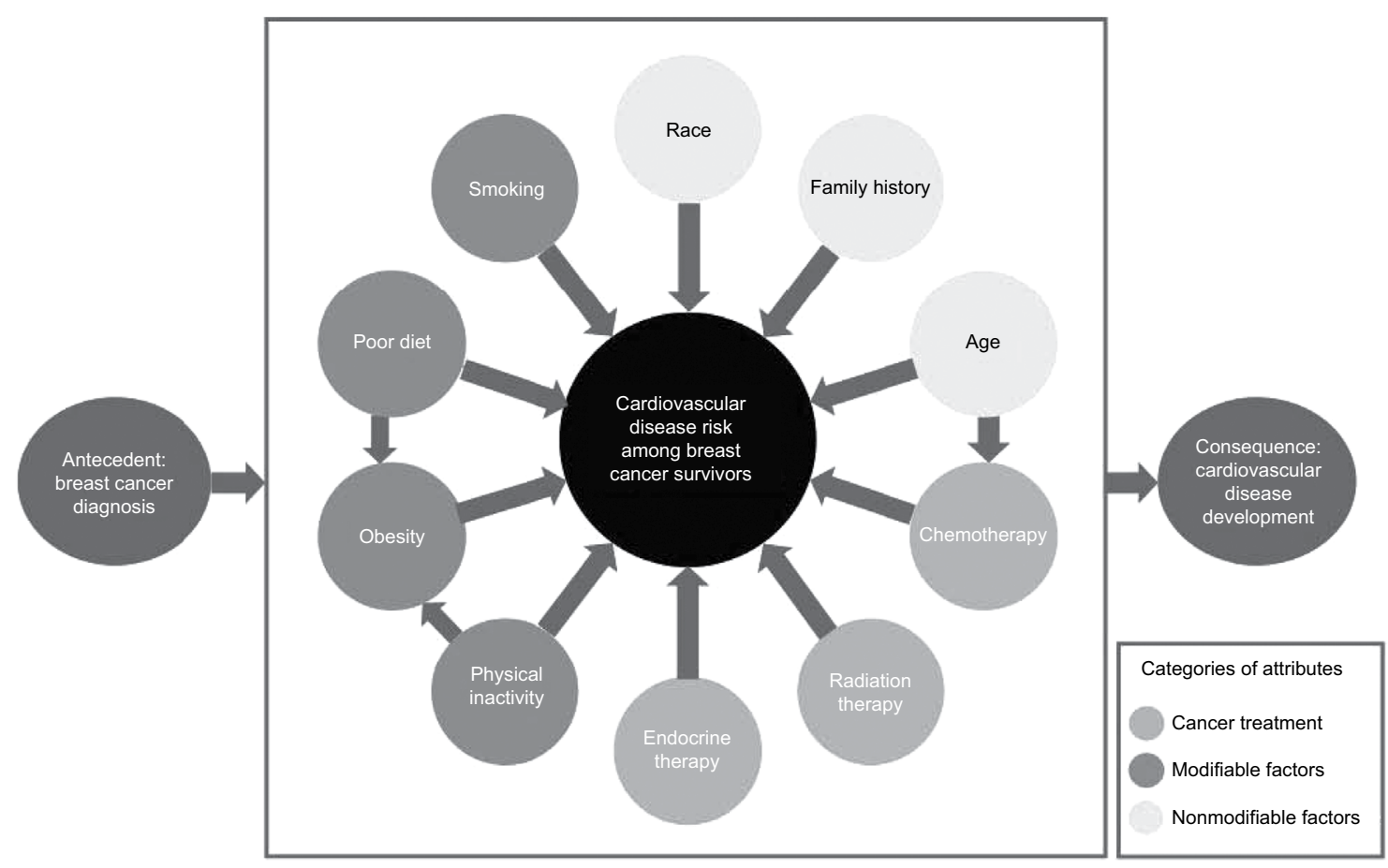

Figure 2 Diagram of cardiovascular disease risk among breast cancer survivors. 
cardiovascular diseases as a result of increased cardiovascular disease risk. Thus, the consequence of this concept is the development of cardiovascular diseases.

\section{Exemplar}

For this concept analysis, the following exemplar is presented. Mrs. Jones is a 65-year-old African American breast cancer survivor. She was diagnosed with breast cancer 12 years ago, and prior to her diagnosis she had no family history of cardiovascular diseases. Yet, she was treated with anthracyclines and taxanes, in addition to radiation to the left breast. She does not exercise, eats a diet high in cholesterol, has a body mass index of $30 \mathrm{~kg} / \mathrm{m}^{2}$, and has smoked one pack per day for the past 30 years. Her older age, race, physical inactivity, poor diet, obesity, smoking history, and cancer treatment contribute to an increased cardiovascular disease risk.

\section{Hypotheses and implications}

The final step in Rodgers' evolutionary concept analysis method is to identify hypotheses and implications. An example of a hypothesis is "breast cancer survivors have a higher cardiovascular disease risk as compared to the general population". Implications for future development include the following: 1) health care providers may need to implement cardiovascular disease risk monitoring at breast cancer diagnosis, during treatment, and regularly throughout survivorship, 2) breast cancer survivors should be informed of their individual cardiovascular disease risk and encouraged to implement healthy lifestyle choices, and 3) health care providers may consider use of validated risk assessment tools to identify patients at increased cardiovascular disease risk.

\section{Discussion}

This concept analysis identified several risk factors that contribute to an increased cardiovascular disease risk among breast cancer survivors. While several studies explored factors that increase cardiovascular disease risk, there were no reported studies exploring the cumulative effect of cancer treatment and modifiable and nonmodifiable risk factors. Many studies used an epidemiological design and did not involve direct contact with breast cancer survivors. Instead, most studies were retrospective and from secondary sources including medical records, cancer registries, death indexes, and large databases. Investigators were unable to select specific risk factors to explore. Additionally, family history is a known risk factor for cardiovascular disease risk but was not explored in any of the selected studies. A potential reason may be that family history data were not collected. Implications for future research include developing large databases for breast cancer survivors that include all cardiovascular disease risk factors or adding modifiable and nonmodifiable risk factors to existing cancer registries. Essentially, there is a need to conduct prospective studies exploring cardiovascular disease risk among breast cancer survivors.

Studies suggest that cancer survivors, who were at increased cardiovascular disease risk, were not educated on healthy lifestyles to reduce the risk..$^{30,31}$ In particular, no study explored strategies to increase the knowledge of cardiovascular disease risk among breast cancer survivors. Using risk prediction models as a mode to identify and teach breast cancer survivors about cardiovascular disease risk may lead to positive changes in modifiable risk factors.

As breast cancer survivors are living longer, their risk of developing cardiovascular diseases increases. Importantly, preexisting modifiable and nonmodifiable risk factors are coupled with additional risk from breast cancer treatment. Thus, common side effects of breast cancer treatment, such as weight gain and decreased physical activity, can increase cardiovascular disease risk among these women. The majority of breast cancer survivors are over the age of 60 years. ${ }^{45}$ As breast cancer survivors get older, they may experience higher cardiovascular disease risk. Rodgers' evolutionary concept analysis method allows for refinement of concepts. A future concept analysis may be necessary as cancer treatment evolves, survival rate continues to rise, breast cancer survivors get older, and the prevalence of cardiovascular disease increases.

Implementation of survivorship care plans is a method to increase awareness of cardiovascular disease risk among breast cancer survivors. The Institute of Medicine recommends individualized care plans for cancer survivors to summarize the effects and long-term management of cancer. ${ }^{46}$ Using survivorship care plans to promote individualized cardiovascular disease risk follow-up (stratified by the cancer treatment received and personal modifiable and nonmodifiable risk factors) may stimulate healthy lifestyle choices and provide resources to achieve reduced cardiovascular disease risk. Additionally, survivorship care plans can incorporate recommendations for monitoring and screening of cardiovascular diseases.

\section{Limitations}

Limitations of this concept analysis include exclusion of secondary articles and literature not written in English. Secondary articles, such as meta-analyses, were excluded to get clear data. Reviewing secondary articles may have been beneficial to increase understanding of the concept's attributes. Articles not written in English may have provided further insight on the concept. Another limitation was that 
many studies included multiple cancer types and were not specific to breast cancer survivors.

\section{Conclusion}

Cardiovascular disease risk among breast cancer survivors comprises three attributes: 1) cancer treatment risk factors, 2) modifiable risk factors, and 3) nonmodifiable risk factors. Survivors are likely to have complex health needs including the potential for development of long-term side effects from cancer treatment. Without stratified screening and monitoring of cardiovascular disease risk, survivors may unknowingly live with asymptomatic cardiovascular diseases. Nurses can educate breast cancer survivors about cardiovascular disease risk and empower patients to implement healthy lifestyle changes.

Several gaps in the literature were identified. An interdisciplinary team of researchers and clinicians should consider collaborating to conduct prospective studies of cardiovascular disease risk among breast cancer survivors and reduce paucity of literature in this area. Further, health care providers may consider use of survivorship care plans to tailor cardiovascular disease risk management. Lifestyle changes should be encouraged to reduce overall cardiovascular disease risk. Interdisciplinary team members must work in collaboration with breast cancer survivors to mitigate cardiovascular disease risk, which may potentially extend and improve the quality of life.

\section{Acknowledgments}

JBV was supported by doctoral study funding through the Robert Wood Johnson Future of Nursing Scholars Program (grant \#72592), Susan G. Komen Graduate Training in Disparities Research Grant (GTDR-15329376), and UAB School of Nursing Doctoral Scholarship. The authors would like to acknowledge Jennifer Frank, PhD, for editorial support of this paper.

\section{Disclosure}

The authors report no conflicts of interest in this work.

\section{References}

1. CDC. Leading Causes of Death. Atlanta: Center for Disease Control and Prevention; 2013. [updated 2016 October 7]. Available from: http:// www.cdc.gov/nchs/fastats/leading-causes-of-death.htm. Accessed July 22,2016

2. Lloyd-Jones DM, Hong Y, Labarthe D, et al; American Heart Association Strategic Planning Task Force and Statistics Committee. Defining and setting national goals for cardiovascular health promotion and disease reduction: the American Heart Association's strategic Impact Goal through 2020 and beyond. Circulation. 2010;121(4):586-613.

3. Appel JM, Zerahn B, Møller S, et al. Long-term heart function after adjuvant epirubicin chemotherapy for breast cancer. Acta Oncol. 2012; 51(8):1054-1061.
4. Doyle JJ, Neugut AI, Jacobson JS, Grann VR, Hershman DL. Chemotherapy and cardiotoxicity in older breast cancer patients: a populationbased study. J Clin Oncol. 2005;23(34):8597-8605.

5. Ganz PA, Hussey MA, Moinpour CM, et al. Late cardiac effects of adjuvant chemotherapy in breast cancer survivors treated on Southwest Oncology Group protocol s8897. J Clin Oncol. 2008;26(8):1223-1230.

6. American Cancer Society. Cancer Treatment \& Survivorship: Facts \& Figures 2016-2017. Atlanta, GA: American Cancer Society; 2016.

7. Surveillance, Epidemiology, and End Results. SEER Stat Fact Sheets: Female Breast Cancer. Maryland: National Cancer Institute. Available from: http://seer.cancer.gov/statfacts/html/breast.html. Accessed July 22, 2016.

8. Patnaik JL, Byers T, DiGuiseppi C, Dabelea D, Denberg TD. Cardiovascular disease competes with breast cancer as the leading cause of death for older females diagnosed with breast cancer: a retrospective cohort study. Breast Cancer Res. 2011;13(3):R64.

9. Runowicz CD, Leach CR, Henry NL, et al. American Cancer Society/ American Society of Clinical Oncology breast cancer survivorship care guideline. J Clin Oncol. 2016;34(6):611-635.

10. National Comprehensive Cancer Network. NCCN Clinical Practice Guidelines in Oncology: Survivorship. Fort Washington, PA: National Comprehensive Cancer Network, Inc.; 2015. Available from: http:// www.nccn.org/professionals/physician_gls/pdf/survivorship.pdf. Accessed July 22, 2016.

11. Bovelli D, Plataniotis G, Roila F; ESMO Guidelines Working Group. Cardiotoxicity of chemotherapeutic agents and radiotherapy-related heart disease: ESMO Clinical Practice Guidelines. Ann Oncol. 2010; 21(Suppl 5):v277-v282.

12. Rodgers BL. 2000. Concept analysis: an evolutionary view. In: Rodgers BL, Knafl KA, editors. Concept Development in Nursing: Foundations, Techniques and Applications. Philadelphia: Saunders; 2000: 77-102.

13. McEwan M, Wills EM. Theoretical Basis for Nursing. 4th ed. Philadelphia, PA: Wolters Kluwer Health/Lippincott Williams \& Wilkins; 2014.

14. Boekel NB, Schaapveld M, Gietema JA, et al. Cardiovascular disease risk in a large, population-based cohort of breast cancer survivors. Int J Radiat Oncol Biol Phys. 2016;94(5):1061-1072.

15. Boerman LM, Berendsen AJ, van der Meer P, Maduro JH, Berger MY, de Bock GH. Long-term follow-up for cardiovascular disease after chemotherapy and/or radiotherapy for breast cancer in an unselected population. Support Care Cancer. 2014;22(7):1949-1958.

16. Bowles EJ, Wellman R, Feigelson HS, et al; Pharmacovigilance Study Team. Risk of heart failure in breast cancer patients after anthracycline and trastuzumab treatment: a retrospective cohort study. J Natl Cancer Inst. 2012;104(17):1293-1305.

17. Bradshaw PT, Stevens J, Khankari N, Teitelbaum SL, Neugut AI, Gammon MD. Cardiovascular disease mortality among breast cancer survivors. Epidemiology. 2016;27(1):6-13.

18. Armenian SH, Xu L, Ky B, et al. Cardiovascular disease among survivors of adult-onset cancer: a community-based retrospective cohort study. J Clin Oncol. 2016;34(10):1122-1130.

19. Darby SC, Ewertz M, McGale P, et al. Risk of ischemic heart disease in women after radiotherapy for breast cancer. $N$ Engl J Med. 2013; 368(11):987-998.

20. Ezaz G, Long JB, Gross CP, Chen J. Risk prediction model for heart failure and cardiomyopathy after adjuvant trastuzumab therapy for breast cancer. J Am Heart Assoc. 2014;3(1):e000472.

21. Haque R, Prout M, Geiger AM, et al. Comorbidities and cardiovascular disease risk in older breast cancer survivors. Am J Manag Care. 2014; 20(1):86-92.

22. Harris EE, Correa C, Hwang WT, et al. Late cardiac mortality and morbidity in early-stage breast cancer patients after breast-conservation treatment. J Clin Oncol. 2006;24(25):4100-4106.

23. Highland KB, Hurtado-de-Mendoza A, Stanton CA, Dash C, Sheppard VB. Risk-reduction opportunities in breast cancer survivors: capitalizing on teachable moments. Support Care Cancer. 2015;23(4):933-941. 
24. Hooning MJ, Botma A, Aleman BM, et al. Long-term risk of cardiovascular disease in 10-year survivors of breast cancer. J Natl Cancer Inst. 2007;99(5):365-375.

25. Jordan JH, Thwin SS, Lash TL, et al. Incident comorbidities and allcause mortality among 5-year survivors of Stage I and II breast cancer diagnosed at age 65 or older: a prospective-matched cohort study. Breast Cancer Res Treat. 2014;146(2):401-409.

26. Leung HW, Chan AL, Muo CH. Late cardiac morbidity of adjuvant radiotherapy for early breast cancer - a population-based study. J Cardiol. 2016;67(6):567-571.

27. Nichols HB, Trentham-Dietz A, Egan KM, et al. Body mass index before and after breast cancer diagnosis: associations with all-cause, breast cancer, and cardiovascular disease mortality. Cancer Epidemiol Biomarkers Prev. 2009;18(5):1403-1409.

28. Obi N, Gornyk D, Heinz J, et al. Determinants of newly diagnosed comorbidities among breast cancer survivors. J Cancer Surviv. 2014;8(3):384-393.

29. Qin A, Thompson CL, Silverman P. Predictors of late-onset heart failure in breast cancer patients treated with doxorubicin. J Cancer Surviv. 2015;9(2):252-259.

30. Enright KA, Krzyzanowska MK. Control of cardiovascular risk factors among adult cancer survivors: a population-based survey. Cancer Causes Control. 2010;21(11):1867-1874.

31. Weaver KE, Foraker RE, Alfano CM, et al. Cardiovascular risk factors among long-term survivors of breast, prostate, colorectal, and gynecologic cancers: a gap in survivorship care? J Cancer Surviv. 2013;7(2):253-261.

32. Burnett D, Kluding P, Porter C, Fabian C, Klemp J. Cardiorespiratory fitness in breast cancer survivors. Springerplus. 2013;2(1):68.

33. Cantarero-Villanueva I, Galiano-Castillo N, Fernandez-Lao C, et al. The influence of body mass index on survival in breast cancer patients. Clin Breast Cancer. 2015;15(2):e117-e123.

34. Caro-Morán E, Fernández-Lao C, Galiano-Castillo N, CantareroVillanueva I, Arroyo-Morales M, Díaz-Rodríguez L. Heart rate variability in breast cancer survivors after the first year of treatments: a case-controlled study. Biol Res Nurs. 2016;18(1):43-49.

35. de Azambuja E, Ameye L, Diaz M, et al. Cardiac assessment of early breast cancer patients 18 years after treatment with cyclophosphamide-, methotrexate-, fluorouracil- or epirubicin-based chemotherapy. Eur J Cancer. 2015;51(17):2517-2524.
36. Gallicchio L, Calhoun C, Helzlsouer K. Effect of aromatase inhibitor therapy on the cardiovascular health of black and white breast cancer patients. Clin Breast Cancer. 2016;16(3):e23-e31.

37. Ho E, Brown A, Barrett P, et al. Subclinical anthracycline- and trastuzumab-induced cardiotoxicity in the long-term follow-up of asymptomatic breast cancer survivors: a speckle tracking echocardiographic study. Heart. 2010;96(9):701-707.

38. Inanc MT, Karadavut S, Aytekin M, et al. The relationship between plasma hyaluronan levels and anthracycline-related cardiotoxicity in breast cancer patients. Int J Cardiol. 2016;218:246-251.

39. Jones LW, Haykowsky M, Peddle CJ, et al. Cardiovascular risk profile of patients with HER2/neu-positive breast cancer treated with anthracycline-taxane-containing adjuvant chemotherapy and/or trastuzumab. Cancer Epidemiol Biomarkers Prev. 2007;16(5):1026-1031.

40. Murtagh G, Lyons T, O'Connell E, et al. Late cardiac effects of chemotherapy in breast cancer survivors treated with adjuvant doxorubicin: 10-year follow-up. Breast Cancer Res Treat. 2016;156(3):501-506.

41. National Heart, Lung, and Blood Institute. How does smoking affect the heart and blood vessels? Maryland: National Institute of Health. [updated June 22, 2016]. Available from: http://www.nhlbi.nih.gov/ health/health-topics/topics/smo/. Accessed July 22, 2016.

42. National Heart, Lung, and Blood Institute. What are the risk factors for heart disease? Maryland: National Institute of Health. [updated July 10, 2014]. Available from: http://www.nhlbi.nih.gov/health/ educational/hearttruth/lower-risk/risk-factors.htm. Accessed July 22, 2016.

43. World Heart Federation. Family history. Available from: http:/www. world-heart-federation.org/cardiovascular-health/cardiovasculardisease-risk-factors/family-history/. Accessed July 22, 2016.

44. National Coalition for Cancer Survivorship. NCCS: Our Mission. Available from: http://www.canceradvocacy.org/about-us/our-mission/. Accessed July 22, 2016.

45. DeSantis CE, Lin CC, Mariotto AB, et al. Cancer treatment and survivorship statistics, 2014. CA Cancer J Clin. 2014;64(4):252-271.

46. Institute of Medicine. Implementing Cancer Survivorship Care Planning. Washington, DC: The National Academies Press; 2007.

47. Moher D, Liberati A, Tetzlaff J, Altman, DG; PRISMA Group. Preferred reporting items for systematic reviews and meta-analyses: the PRISMA statement. PLoS Med. 2009;6(7):e1000097.
Nursing: Research and Reviews

\section{Publish your work in this journal}

Nursing: Research and Reviews is an international, peer-reviewed, open access journal publishing original research, reports, reviews and commentaries on all aspects of nursing and patient care. These include patient education and counseling, ethics, management and organizational issues, diagnostics and prescribing, health outcomes, economics and
Dovepress

resource management, improving patient safety in all settings. The manuscript management system is completely online and includes a very quick and fair peer-review system. Visit http://www.dovepress. com/testimonials.php to read real quotes from published authors. 